\title{
Discussion to Papers of Wicks and Dinsdale, and Forner
}

DR E. BERARD (France). I will ask the two first speakers. Have you tried to perform your investigations with and without any abdominal contention?

Dr P. Dollfus (France). My question is quite close to his, to the second speaker, Dr Forner. How spastic were your tetraplegics, because now we have been trying to diminish unfortunately temporary spasticity in tetraplegics and we've found variations of vital capacity in the same patient if you suppress partially his spasticity. It goes up.

DR G. HAHN (Canada). Dr Forner, I think it is true that maximum flow rate measurements are less afferent dependent than simple peak expiratory flow rate measurements, at least in my experience with asthmatic children. What I'd like to know from your work which I found exciting to listen to, do you think that in a clinical setting where one doesn't have access to a pulmonary function laboratory or machinery to do the eloquent flow rates that you measured, it is adequate to use a simple Wright peak expiratory flow measurement to get pretty equivalent data as far as comparison to maximum expiratory flow rates is concerned.

DR FORNER. I think that at the moment discussion of that very clinical usefulness is not very important. At the moment we are doing a more like investigation. I don't know whether it will be really very important in the future to record. Also, we don't know the difference between the early stages and the later stages having done tests only in the later stages of paraplegia. All the patients have been studied without corsets so without any abdominal contention, all but one were spastics. How much spasticity it is very difficult to assess. I don't think they were spastic. They are complete tetraplegics with moderate spasticity I would say. 